\title{
The impact of butylphthalide on the hypothalamus-pituitary-adrenal axis of patients suffering from cerebral infarction in the basal ganglia
}

Huiyan Niu, Zhanqiang Zhang, Hai Wang, Huifang Wang, Jinyan Zhang, Chunbo Li, Liang Zhao

Neurology Department, Zhuozhou City Hospital of Hebei Province, 072750, China

Type of article: Original

\begin{abstract}
Background: Butylphthalide sodium chloride injection for patients with acute cerebral infarction has a certain effect. Although there are several proposed mechanisms of drug action, no related research on improving the inflammatory cytokines that regulate the body's immune system through the hypothalamus-pituitary-adrenal axis has been published.

Objective: To determine the impact of butylphthalide and sodium chloride injection on the hypothalamuspituitary-adrenal (HPA) axis after acute cerebral infarction in the basal ganglia.

Methods: Patients were randomly divided into treatment and control groups; the treatment group received intravenous drips of butylphthalide, while the control group did not. The levels of adrenocorticotropic hormone (ACTH) and cortisol (COR), along with the National Institutes of Health Stroke Scale (NIHSS) scores of both groups were detected using the radioimmunoassay method. This was done at regular intervals after cerebral infarction in the basal ganglia was detected.

Results: Fourteen days after treatment, the levels of serum ACTH and COR in both groups were higher than normal. The NIHSS score and levels of ACTH and COR of the treatment group were significantly lower than those of the control group $(\mathrm{p}<0.05)$. The data was computed and analyzed using SPSS17.0 software.

Conclusion: Butylphthalide treatment for patients suffering from acute basal ganglia infarction can reduce the adverse effects on the HPA axis, thus improving patient prognosis.

Trial registration: The trial is registered at the Chinese Clinical Trial Registry (www.chictr.org.cn) under the registration number ChiCTR-CPR-15007593.

Funding: This work was supported by the National Twelfth Five-Year Plan for Science and Technology Pillar Program of People's Republic of China [Program No. 14ZF013 and 2011BA108B03]

Keywords: cerebral infarction, ganglia region; hypothalamus-pituitary-adrenal axis; HPA axis; butylphthalide
\end{abstract}

\section{Introduction}

There is increasing interest regarding changes in the hypothalamus-pituitary-adrenal (HPA) axis of patients with acute cerebrovascular disease. The occurrence of cerebral infarction will not only cause damage to nerve function, but also cause endocrine, metabolic, and primarily hypothalamus-pituitary-adrenal (HPA) function changes (1). Studies have shown that the hypothalamus-pituitary-adrenal (HPA) axis of patients with acute cerebral infarction (ACI) may be affected, and in turn, cause hormone secretion disorders (2-5). Such disorders can aggravate the existing disease and result in a variety of complications. This study aims to discover whether administration of certain medications can restore normal HPA axis facilitated hormone secretion, thereby inhibiting exacerbation of disease and reducing incidences of complications. The general objective of this research is to observe the impact of butylphthalide on the HPA axis of patients suffering from cerebral infarction in the basal ganglia. The specific objectives are 1) to determine the level of ACTH and COR on the $1^{\text {st }}$ and $14^{\text {th }}$ day of the treatment, respectively, 2) to determine the NIHSS score on the $1^{\text {st }}, 14^{\text {th }}$, and $30^{\text {th }}$ day after treatment, followed by refinement of results, and

\section{Corresponding author:}

Huiyan Niu, Neurology Department, Zhuozhou City Hospital of Hebei Province, 072750, China.

Email: huiyan_2015@163.com

Received: November 08, 2015, Accepted: January 04, 2016, Published: January 2016

iThenticate screening: January 04, 2016, English editing: January 08, 2016, Quality control: January 10, 2016

(C) 2015 The Authors. This is an open access article under the terms of the Creative Commons Attribution-NonCommercialNoDerivs License, which permits use and distribution in any medium, provided the original work is properly cited, the use is non-commercial and no modifications or adaptations are made. 
analysis of the relationship between hormone secretion and NIHSS score before and after treatment, and 3) to observe the possibility of adverse drug reactions during treatment.

\section{Material and Methods}

\subsection{Selection criteria:}

The followings were set as the selection criteria: 1) meeting the diagnostic standard developed by the Fourth National Conference on Cerebrovascular Disease of China, 2) admission into the hospital within 48 hours of onset, 3 ) exclusion of hemorrhagic cerebrovascular disease, as diagnosed by imaging, 4) an age of onset between 45 and 82 years old, 5) that patients were able to take care of themselves before onset, and 6) exclusion of patients with severe infection, cancer, multiple system dysfunction, or tendency towards bleeding.

\subsection{Study setting, groups, and randomization}

This study selected 84 AHI patients, who were admitted for treatment to the Neurology Department of Zhuozhou City Hospital of Hebei Province between June 2013 and June 2015. They were divided randomly into two groups according to their proximity with regard to NIHSS score. The method to determine the groups was that the total patients involved in this trial were divided into 42 sub-groups with each sub-group consisting of two patients. The patients in each sub-group were similar in age and NIHSS score. All the sub-groups were randomly assigned a group number of 1 to 42. Both of the patients in each sub-group were randomly given a label of A or B. All the patients A in the odd sub-groups and patients B in the even sub-groups were organized into one test group and the remaining patients were organized into the other test group.

\subsection{Interventions}

\subsubsection{Method of administration}

Both groups received treatment after admission, including platelet aggregation inhibition, blood lipid reduction for atherosclerosis prevention, blood circulation and cerebral metabolic improvement, and blood pressure and blood sugar regulation. The experimental group received the additional treatment of intravenous drips of butylphthalide and sodium chloride injection $(25 \mathrm{mg}$, twice a day).

2.3.2. Observation of medical indexes and evaluation of therapeutic efficacy

1) Observation of medical indexes: A radioimmunoassay method was used to test the levels of adrenocorticotropic hormone (ACTH) (normal: 2.2-17.6 pmol/L at 8:00 am) and cortisol (COR) (normal: 210-342 $\mathrm{nmol} / \mathrm{L}$ between 8:00-9:00 am) of venous blood (5 ml collected at 8:00 am). The isolated serum samples were stored in a cryogenic box and measure quantitatively. Serum levels were measured by radioimmunoassay in accordance with the operation manual of the reagents that we used. The reagents were supplied by the Beijing North Institute of Biological Technology.

2) Evaluation of therapeutic efficacy: An efficacy evaluation was made at 1, 14, and 30 days post-treatment, based on the NIHSS score. Efficacy of neurological deficit scored changes were rated as follows: reduced 91\%-100\% (significantly effective), reduced 18\%-19\% (effective), reduced less than 18\% (ineffective), and increased more than 18\% (aggravated). The overall therapeutic efficacy was calculated using the following formula: overall therapeutic efficacy $=$ (No. patients with significantly effective score changes + No. of patients with effectivescore changes) / total patient $\times 100 \%$.

\subsection{Statistical analysis methods}

Data calculation and analysis were performed using SPSS version 17.0 (SPSS Inc., Chicago, Illinois, USA). The data count was expressed as a relative number (\%) or rate (\%), and conducted using the "Chi-square test". Measurement data was expressed as mean \pm standard deviation $(\mathrm{x} \pm \mathrm{s})$; repeated measurement analysis of variance was made, and a $\mathrm{p} \leq 0.05$ was considered as statistically significant.

\subsection{Research ethics}

The ethics committee in the Zhuozhou City Hospital of Hebei Province approved this research. The trial is registered at the Chinese Clinical Trial Registry.

\section{Results}

The experimental group (treated with butylphthalide and sodium chloride injection) consisted of 42 patients, 26 male and 16 female, aged between 45 and 82 years, with a mean of $63.60 \pm 7.24$ years. NIHSS score of 4 to 20 , with an average of $12.12 \pm 4.01$. Conventional treatment group (control group) has 42 patients, 26 male and 18 female; 
aged between 46 and 81 years, with a mean of $63.51 \pm 8.37$ years. NIHSS scores ranged from 4 to 20 , with an average of $11.98 \pm 4.67$. The differences in gender, average age, average NIHSS score, and other data between the two groups were not statistically significant. Acute cerebral infarction in the basal ganglia has proven to cause a significant increase in serum ACTH and COR levels. There was no significant difference in ACTH values $(\mathrm{p}=0.887)$ and COR values $(p=0.694)$ between the two groups before treatment. However, 14 days after the treatment, the ACTH levels of the two groups had decreased, but the decrease in ACTH levels of the experimental group was more significant. Thus, there is a statistically significant difference $(p<0.05)$ between treated and control groups (Table 1 and Table 2). The difference in NIHSS scores of the two groups before treatment was not statistically significant $(p=0.783)$. However, 14 days after treatment, the NIHSS scores of the two groups had decreased, with the experimental group having a higher rate of decrease in NIHSS score than the control group, with statistical significance between the two groups $(\mathrm{p}=0.029)$ (Table 3). In the treatment group, there were 2 cases of ineffectiveness and 1 case of aggravation, with an overall efficiency of $92.86 \%$. In the control group, on the other hand, there were 5 cases of ineffectiveness and 4 cases of aggravation, while the overall efficiency was $78.57 \%$ (Table 4). Thus, the results from the experimental group indicated a significant improvement, compared to those of the control group. After 30 days of treatment, the difference in the NIHSS scores between the two groups was proven to be statistically significant $(p=0.025)$. The two groups of patients were examined after admission. There were 18 cases with elevated blood lipids in the treatment group and 16 cases in the control group. The rest of the indicators were normal. Incidences of hyperlipidemia in both groups were treated on day 14. Both groups had 2 cases with elevated aspartate aminotransferase and alanine aminotransferase after liver therapy, with all the indexes returning to normal after 30 days. The whole course of treatment recorded no adverse reactions or serious side effects.

Table 1. Analysis of ACTH levels

\begin{tabular}{|l|l|l|l|}
\hline Group & Cases & Before treatment $(\mathrm{pmol} / \mathrm{L})$ & On the $14^{\text {th }}$ day $(\mathrm{pmol} / \mathrm{L})$ \\
\hline Treatment group & 42 & $42.78 \pm 8.81$ & $20.19 \pm 7.28$ \\
\hline Control group & 42 & $41.83 \pm 9.37$ & $28.60 \pm 6.98$ \\
$\mathrm{~T}$ & & 0.720 & 4.316 \\
$P$ & & 0.887 & 0.027 \\
\hline
\end{tabular}

Table 2. Analysis of COR levels

\begin{tabular}{|l|l|l|l|}
\hline Group & Cases & Before treatment $(\mathrm{nmol} / \mathrm{L})$ & On the $14^{\text {th }}$ day $(\mathrm{nmol} / \mathrm{L})$ \\
\hline Treatment group & 42 & $568.57 \pm 56.34$ & $386.23 \pm 62.18$ \\
\hline Control group & 42 & $579.14 \pm 51.87$ & $451.42 \pm 58.63$ \\
T & & 0.926 & 7.124 \\
$P$ & & 0.694 & 0.035 \\
\hline
\end{tabular}

Table 3. Analysis of NIHSS score

\begin{tabular}{|l|l|l|l|l|}
\hline Group & Cases & Before treatment & After 14 days of treatment & After 30 days of treatment \\
\hline Treatment group & 42 & $12.12 \pm 4.01$ & $6.78 \pm 3.29$ & $4.15 \pm 3.29$ \\
\hline Control group & 42 & $11.98 \pm 4.67$ & $8.91 \pm 4.14$ & $7.02 \pm 4.16$ \\
T & & -0.721 & 6.728 & 5.253 \\
$P$ & & 0.783 & 0.029 & 0.025 \\
\hline
\end{tabular}

Table 4. Comparison of the total efficiency after 14 days of treatment

\begin{tabular}{|l|l|l|l|l|l|l|}
\hline Group & Cases & Significantly Effective & Effective & Ineffective & Aggravation & Total efficiency \\
\hline $\begin{array}{l}\text { Treatment } \\
\text { group }\end{array}$ & 42 & $19(45.24 \%)$ & $20(47.62 \%)$ & $2(4.76 \%)$ & $1(0.00 \%)$ & $92.86 \%$ \\
\hline Control group & 42 & $18(42.86 \%)$ & $15(35.71 \%)$ & $5(11.90 \%)$ & $4(9.52 \%)$ & $78.57 \%$ \\
\hline
\end{tabular}

\section{Discussion}

The hypothalamus is the central region of neuroendocrine activity and comprises the bottom wall of the third ventricle, infundibulum, gray nodules, mammillary body, etc. The cranial nerve input of the hypothalamus plays a role in almost all activities of the brain, with all nerve inputs centering at the hypothalamus, which acts as the releasing factor pertaining to neuroendocrine. Corticotropin-release facilitated by the hypothalamus acts on the pituitary gland, which in turn secretes adrenocorticotropic hormones that acts on the adrenal glands to release COR. 
COR is involved in a feedback regulation mechanism that acts on the pituitary gland and the hypothalamus. All three then integrate into the neuroendocrine axis, which has important regulatory functions. The HPA axis maintains the stability of the internal environment and stress response, function of which directly affects the body. Under normal physiological conditions, COR secreted by the adrenal glands exhibits negative feedback regulation on the HPA axis. But, under stress conditions, the hypothalamus corticotropin releasing hormone (CRH) is excessively secreted, which further leads to excessive secretion of pituitary ACTH, and eventually leads to COR hypersecretion. Excessive COR causes dysfunction of the HPA axis negative feedback mechanism, leading to continual increase in concentration of hypothalamic $\mathrm{CRH}$, pituitary $\mathrm{ACTH}$, and adrenal $\mathrm{COR}$, which is referred to as continued hyperactivity of the HPA axis, namely HPA axis activation $(6,7)$. When acute cerebral infarction occurs, the intracerebral environment of patients becomes disordered. Since the HPA axis is activated, ACTH and plasma or urine COR increases after an acute stroke (4). The concentration of ACTH or COR in an acute ischemic stroke is related to the degree of severity in the stroke, defined as a larger infarcted area and/or poor prognosis, including death (5). In clinical settings, basal ganglia infarction is the most common. The results of this study demonstrate that when acute cerebral infarction occurs, serum ACTH and COR are significantly higher than normal. After 14 days of butylphthalide and sodium chloride treatment, ACTH and COR of both groups decreased, with a more significant decrease observed in the experimental group $(\mathrm{p}<0.05)$.

The results indicate that during the acute stage of cerebrovascular disease, the HPA axis is directly related to the emergency state after brain injury. ACTH and COR levels are significantly decreased after butylphthalide treatment, positively correlated with the NIHSS score. A previous study found that after correction of vascular risk factors, age and the NIHSS score, COR is associated with mortality in stroke patients, but has no significant correlation with functional outcomes (8). Catecholamines and COR increase simultaneously after a stroke, and these two factors can increase the risk of infection (9). This is consistent with the immune suppression concept induced by the brain after a stroke, as the release of immunosuppressants is partly due to a post-stoke higher susceptibility to infection caused by this systemic stress response (10). At the same time, this study also found that larger infarcted areas lead to higher ACTH and COR values, but does not positively correlate with NIHSS scores, while higher blood sugar level leads to higher ACTH and COR values, which is positively correlated with NIHSS scores as it increases the body's tolerance to hypoxia (11). This phenomenon may be related to direct stimulation of pituitary-adrenal cortex function: the adrenal cortex is stimulated to increase secretion of glucocorticoids. The initial part of the stimulus affects the anterior pituitary, therefore, the plasma COR and ACTH levels increase in parallel, indicating a HPA axis disorder, which is a serious condition. Application of butylphthalide and sodium chloride injections can adjust the emergency state of the HPA axis and improve the NIHSS score. Regulating the HPA axis with butylphthalide can improve immunity, reduce the impact of inflammation related factors on brain tissue and associated blood vessels, reduce the risk of infection, promote blood supply to ischemic areas, and improve brain metabolism, thereby improving the prognosis and lowering the NIHSS score, which promotes clinical recovery. During the entire course of treatment, there were no adverse reactions. Both groups of patients had two cases of elevated aminotransferases, which become normal after treatment, taking into consideration the correlation with application of atorvastatin calcium. Regarding the study limitations, in this study, only the 30 day NIHSS score was evaluated. The decrease of the treatment group is statistically significant compared to that of the control group, the ACTH and COR values after 30 days were not perfected, although ACTH and COR values gradually declined, but it is not clear when they fully returns to normal.

\section{Conclusions}

In summary, in cases of acute cerebral infarction, HPA axis disorders will cause a rapid increase of hormone secretion. Intravenous drips of butylphthalide and sodium chloride injections can adjust the HPA axis, improve the body's immunity, reduce infection and other complications, and are an effective brain protection treatment.

\section{Acknowledgments:}

The clinical trial was done in the in the Neurology Department of Zhuozhou City Hospital of Hebei Province. China. The authors would like to express their deepest appreciation to the department and all those who provided them with the opportunity to complete this trial.

\section{Clinical trial registration:}

The trial is registered at the Chinese Clinical Trial Registry (www.chictr.org.cn) with registration number ChiCTRCPR-15007593. 


\section{Funding:}

This work was supported by the National Twelfth Five-Year Plan for Science and Technology Pillar Program of People's Republic of China [Program No. 14ZF013 and 2011BA108B03].

\section{Conflict of Interest:}

There is no conflict of interest to be declared.

Authors' contributions:

All authors contributed to this project and article equally. All authors read and approved the final manuscript.

\section{References}

1) Wang CS, Xie YY. Research status of hypothalamus-pituitary-target gland axis under acute cerebrovascular disease. J Apoplexy Nerv Dis. 1994;11(5):316-8. Chinese. Available from: http://zfsj.cbpt.cnki.net/WKC3/WebPublication/paperDigest.aspx?paperID=C9DCEB81 -82B7-4209-9319D5BE53952860

2) Ouyang TW, Zan JF. Study on changes of plasma stress hormones of hypothalamus-pituitary-adrenal axis and pancreatic hormones in acute cerebral infarction. J Radioimmunol. 2000;13(4):196-8. Chinese. Available from: http://d.wanfangdata.com.cn/Periodical/fsmyxzz200004002

3) Ren M. The interaction between cerebral infarction and hypothamus-pituitary-adrenal axis [dissertation]. [Beijing]: China People's Liberation Army Postgraduate Medical School; 1996.

4) Christensen H, Boysen G, Johannesen HH. Serum-cortisol reflects severity and mortality in acute stroke. J Neurol Sci. 2004 Feb;217(2):175-80. DOI: 10.1016/j.jns.2003.09.013, PMID: 14706221

5) Anne M, Juha K, Timo M, Mikko T, Olli V, Kyösti S, Heikki H, Vilho M. Neurohormonal activation in ischemic stroke: effects of acute phase disturbances on long-term mortality. Curr Neurovasc Rec. 2007 Aug;4(3):170-5. DOI: 10.2174/156720207781387169, PMID: 17691970

6) Sun HS, Zheng WQ, Liu W, Tan LL, Fu JH. Effect of acute or chronic stress on the hypothalamicpituitary-adrenal axia function in high calorie diet fed rats. Anhui Med Pharm J. 2010;14 (1):17-9. Chinese. Available from: http://mall.cnki.net/magazine/magadetail/AHYY201001.htm

7) Jia BH, Li ZG, Shi YJ, Fei YP, Zhang HM, Tu Y. Effect of electroacupuncture on changes of behavior and some related hormones of hypothalamus-pituitary-adrenal axis in chronic stress model rats. Acupunct Res. 2004;29(04):252-6. Chinese. Available from: http://www.zhenciyanjiu.cn/WKA/WebPublication/paperDigest.aspx?paperID=474F84B7-48A7-437A9561-242FEB26193B

8) Neidert S, Katan M, Schuetz P, Fluri F, Ernst A, Bingisser R, Kappos L, Engelter ST, Steck A, Müller B, Christ-Crain M. Anterior pituitary axis hormones and outcome in acute ischaemic stroke. J Intern Med. 2011 Apr;269(4):420-32. DOI: 10.1111/j.1365-2796.2010. 02327, PMID: 21205022

9) Urra X, Cervera A, Obach V, Climent N, Planas AM, Chamorro A. Monocytes are major players in the prognosis and risk of infection after acute stroke. Stroke. 2009 Apr;40(4):1262-8. DOI: 10.1161/STROKEAHA.108.532085, PMID: 19164783

10) Klehmet J, Harms H, Richter M, Prass K, Volk HD, Dirnagl U, Meisel A, Meisel C. Stroke-induced immunodepression and post-stroke infections: lessons from the preventive antibacterial therapy in stroke trial. Neuroscience. 2009 Feb 6;158(3):1184-93. Doi: 10.1016/j.neuroscience.2008.07.044, PMID: 18722511

11) Tu HH, Zhang RX, Jia ZP, Zhang X, Lan JG, Xue X. Effect of three-recovery capsule on glucose metabolism and HPA axis function of hypoxia mice. Pharm J Chin People's Liberation Army. 2009;25(3):216-20. Chinese. Available from: http://www.jfjyxxb.cn/Magazine/Show.aspx?Id=97506 